\section{OPEN ACCESS}

Edited by:

Hamid R. Sohrabi,

Macquarie University, Australia

Reviewed by:

Elizabeth Rochon,

University of Toronto, Canada

Argye Hillis,

Johns Hopkins Medicine,

United States

*Correspondence:

Jason D. Warren

jason.warren@ucl.ac.uk

†Joint senior authors.

Specialty section:

This article was submitted

to Neurodegeneration,

a section of the journal

Frontiers in Neurology

Received: 04 September 2017 Accepted: 02 November 2017 Published: 16 November 2017

Citation:

Marshall CR, Hardy CJD, Russell LL, Clark CN, Dick KM, Brotherhood EV, Bond RL, Mummery CJ, Schott JM, Rohrer JD, Kilner JM and Warren JD

(2017) Impaired Interoceptive Accuracy in Semantic Variant Primary Progressive Aphasia.

Front. Neurol. 8:610. doi: 10.3389/fneur.2017.00610

\title{
Impaired Interoceptive Accuracy in Semantic Variant Primary Progressive Aphasia
}

\author{
Charles R. Marshall ${ }^{1,2}$, Chris J. D. Hardy ${ }^{1}$, Lucy L. Russell', Camilla N. Clark', \\ Katrina M. Dick ${ }^{1}$, Emilie V. Brotherhood ${ }^{1}$, Rebecca L. Bond ${ }^{1}$, Catherine J. Mummery ${ }^{1}$, \\ Jonathan M. Schott ${ }^{1}$, Jonathan D. Rohrer ${ }^{1}$, James M. Kilner ${ }^{2+}$ and Jason D. Warren ${ }^{1 *+}$ \\ ${ }^{1}$ Dementia Research Centre, Department of Neurodegenerative Disease, London, United Kingdom, ${ }^{2}$ Sobell Department of \\ Motor Neuroscience and Movement Disorders, Institute of Neurology, University College London, London, United Kingdom
}

Background: Interoception (the perception of internal bodily sensations) is strongly linked to emotional experience and sensitivity to the emotions of others in healthy subjects. Interoceptive impairment may contribute to the profound socioemotional symptoms that characterize frontotemporal dementia (FTD) syndromes, but remains poorly defined.

Methods: Patients representing all major FTD syndromes and healthy age-matched controls performed a heartbeat counting task as a measure of interoceptive accuracy. In addition, patients had volumetric MRI for voxel-based morphometric analysis, and their caregivers completed a questionnaire assessing patients' daily-life sensitivity to the emotions of others.

Results: Interoceptive accuracy was impaired in patients with semantic variant primary progressive aphasia relative to healthy age-matched individuals, but not in behavioral variant frontotemporal dementia and nonfluent variant primary progressive aphasia. Impaired interoceptive accuracy correlated with reduced daily-life emotional sensitivity across the patient cohort, and with atrophy of right insula, cingulate, and amygdala on voxel-based morphometry in the impaired semantic variant group, delineating a network previously shown to support interoceptive processing in the healthy brain.

Conclusion: Interoception is a promising novel paradigm for defining mechanisms of reduced emotional reactivity, empathy, and self-awareness in neurodegenerative syndromes and may yield objective measures for these complex symptoms.

Keywords: interoception, autonomic, cardiac, empathy, primary progressive aphasia, frontotemporal dementia

\section{INTRODUCTION}

Interoception (the ability to sense one's internal physiological states) is closely linked to emotional experience (1) and can be measured using awareness of one's heartbeat as a surrogate for interoceptive sensitivity $(2,3)$. According to recent interoceptive inference formulations, hierarchically organized brain networks compare afferent interoceptive information with predictions about bodily states, with prediction errors activating autonomic reflexes or motivating actions to maintain homeostasis (4). At lower hierarchical levels, these relate to direct physiological homeostasis, such as maintaining blood oxygen and glucose levels. Coherent representations of the physiological 
state of one's body are important determinants of subjective feeling states (5), and those with weaker interoception are less able to identify and describe their own emotions (6). At higher hierarchical levels, inferences about more complex causes of physiological perturbations can be made, such as the autonomic changes induced by the emotions of others. Interoception is therefore hypothesized to play a key role in empathy and theory of mind (7). This is borne out by evidence showing that interoceptive ability predicts both sensitivity to the emotions of others and performance on emotional theory of mind tasks $(8,9)$. Empathy has been correlated with the magnitude of heartbeatevoked potentials, and both cognitive and neural responses to the emotions of others are influenced by stimulus timing within the cardiac cycle (10-12).

Interoceptive signals and exteroceptive information from the environment are integrated in a reciprocal manner, with diminished interoception tending to promote greater environmental dependency, and vice versa. Those with less interoceptive ability are more susceptible to exteroceptive signals that alter perception of body ownership (13), while inducing the illusion of decreased body ownership reduces both the amplitude of heartbeat-evoked potentials (14) and the ability to cognitively detect signals arising from the heart (15). Interoception is therefore likely to play a key role in generating a coherent sense of the bodily self. The reciprocal relationship between interoception and exteroception has also been demonstrated in perceptual decision-making, with interoceptive arousal limiting the influence of exteroceptive sensory noise on confidence (16). Interoception entails dissociable cognitive dimensions, interoceptive accuracy (objective reporting) supporting awareness (confidence in interoceptive judgments) (3). Interoceptive sensitivity is mediated principally by cingulate and insula (17) under the influence of amygdala (18). Together, these structures constitute a network engaged in both interoception and emotion processing (5).

Interoception has been hypothesized to be a factor mediating changes in emotional sensitivity in normal aging (19). Different dimensions of interoception-accuracy and awareness-might be separately targeted by brain disease. One leading candidate, on clinical and neuroanatomical grounds, is the group of neurodegenerative diseases comprising frontotemporal dementia (FTD). This heterogeneous entity comprises three major clinicoanatomical syndromes: behavioral variant frontotemporal dementia (bvFTD), semantic variant primary progressive aphasia (svPPA), and nonfluent variant primary progressive aphasia (nfvPPA). All three syndromes profoundly disrupt emotional and physiological reactivity (20-22), producing complex neuropsychiatric symptoms such as loss of empathy and altered bodily awareness $(23,24)$. These symptoms are of key clinical relevance but remain difficult to measure and poorly understood (25). Impaired interoception is a plausible mechanism that may link neurodegeneration to socioemotional phenotypes in FTD (26). However, interoceptive processing has not been studied systematically in the FTD syndromic spectrum nor specifically related to reduced emotional awareness in particular FTD syndromes and to underlying neuroanatomical substrates (26).

Here, we used heartbeat counting to assess interoceptive accuracy in canonical FTD syndromes (svPPA, bvFTD, and
nfvPPA) versus healthy older individuals. We related patients' interoceptive accuracy both to a clinical index of emotional sensitivity and to regional gray matter on voxel-based morphometry (VBM). As all syndromes within the FTD spectrum are associated with socioemotional deficits and insular atrophy, some degree of impaired interoception leading to abnormal emotional awareness is anticipated across the FTD spectrum. However, among FTD syndromes, svPPA in particular has been linked to abnormally heightened responsiveness to exteroceptive stimuli (24), altered bodily awareness, and an impoverished concept of self (27). The associations between interoception, exteroception, body ownership, and sense of self identified in the healthy brain (13-16) suggest that reduced interoceptive accuracy may be a core feature of svPPA and disproportionately severe in this syndrome relative to other FTD syndromes. Moreover, incorporation of interoceptive information into emotional judgments has been shown to depend on the amygdala, which is particularly severely affected in svPPA $(18,28)$. This further suggests a brain mechanism that could link reduced interoceptive accuracy to loss of emotional sensitivity in this syndrome. We therefore hypothesized that all FTD syndromes would be associated with a degree of impaired interoception leading to reduced emotional sensitivity, but that svPPA would be associated with a particularly severe deficit of interoceptive accuracy, based on the specific psychophysiological profile of this syndrome and linked to grey matter loss in a frontotemporal network including amygdala.

\section{MATERIALS AND METHODS}

\section{Participants}

Thirty-two consecutive patients fulfilling consensus criteria for a syndrome of FTD $(29,30)$ (16 bvFTD, 7 svPPA, and 9 nfvPPA) and 19 age-matched healthy individuals [overall 51 participants, mean age 67.6 years (range 51-84), 22 females] participated. No participant had a history of cardiac arrhythmia, clinical depression, or anxiety disorder. Neuropsychological assessment and MR brain imaging corroborated the syndromic diagnosis in all patients. Clinical, demographic, and neuropsychological characteristics of all participants are summarized in Table 1. Participant groups did not differ significantly in age or gender, symptom duration, or use of antihypertensive medication; no participant was taking cardiac rate-limiting medication. The study was approved by the local ethics committee and all participants gave informed consent.

\section{Heartbeat Counting Task}

We adapted a previously described heartbeat counting task as a measure of interoceptive accuracy $(2,3)$. Participants were asked to try to identify their heartbeats by "listening to their body" (rather than feeling their pulse) and were first familiarized with the paradigm to ensure they understood the task. ECG was recorded continuously from electrodes placed over the right clavicle and left iliac crest. During the experiment, the number of sensed beats was reported for four epochs of variable duration $(25,35$, 45 , and $100 \mathrm{~s}$ ) signaled by start and stop tones and presented in 
TABLE 1 | Clinical and neuropsychological characteristics of participant groups.

\begin{tabular}{|c|c|c|c|c|}
\hline Characteristic & Controls & bvFTD & svPPA & nfvPPA \\
\hline \multicolumn{5}{|l|}{ Demographic and clinical } \\
\hline No $(m: f)$ & 8:11 & $13: 3$ & $5: 2$ & $4: 5$ \\
\hline Age (years) & $68.8(5.5)$ & $65.8(7.3)$ & $65.9(7.4)$ & $69.6(6.5)$ \\
\hline Handedness (R:L:A) & $17: 1: 1$ & 15:1:0 & 7:0:0 & $7: 2: 0$ \\
\hline MMSE $(/ 30)$ & $29.6(0.6)$ & $24.6(4.5)^{\mathrm{a}}$ & $22.6(5.8)^{\mathrm{a}}$ & $23.7(6.0)^{\mathrm{a}}$ \\
\hline Duration (years) & N/A & $7.6(4.7)$ & $4.4(2.0)$ & $4.6(2.2)$ \\
\hline EX & N/A & $5.4(4.7)^{d}$ & $9.5(2.3)^{d}$ & $20.0(7.6)$ \\
\hline Mean heart rate & $69.5(10.2)^{d}$ & $72.5(12.9)$ & $69.7(5.2)^{d}$ & $85.5(17.1)$ \\
\hline \multicolumn{5}{|l|}{ Neuropsychological } \\
\hline \multicolumn{5}{|l|}{ General intellect } \\
\hline WASI verbal IQ & $125.4(7.0)$ & $86.4(22.4)^{\mathrm{a}}$ & $78.6(20.4)^{a}$ & $80.0(17.3)^{\mathrm{a}}$ \\
\hline WASI performance IQ & $125.1(9.7)$ & $102.44(21.4)^{\mathrm{a}}$ & $112.3(20.1)$ & $98.8(21.5)^{\mathrm{a}}$ \\
\hline \multicolumn{5}{|l|}{ Episodic memory } \\
\hline RMT words (/50) & $49.3(0.9)$ & $36.2(8.0)^{a}$ & $30.3(6.9)^{\mathrm{a}, \mathrm{d}}$ & $41.4(9.5)^{\mathrm{a}}$ \\
\hline RMT faces (/50) & $44.7(3.7)$ & $34.0(7.6)^{a}$ & $32.7(6.4)^{\mathrm{a}}$ & $39.5(6.6)$ \\
\hline Camden PAL (/24) & $20.3(3.5)$ & $10.5(7.5)^{\mathrm{a}}$ & $2.7(4.2)^{a, b, d}$ & $16.3(7.8)$ \\
\hline \multicolumn{5}{|l|}{ Executive skills } \\
\hline WASI block design (/71) & $46.0(10.1)$ & $32.6(19.2)$ & $41.6(19.0)$ & $25.1(19.7)^{\mathrm{a}}$ \\
\hline WASI matrices (/32) & $26.6(4.1)$ & $17.8(9.4)^{\mathrm{a}}$ & $21.7(8.5)$ & $17.4(9.0)^{\mathrm{a}}$ \\
\hline WMS-R digit span forward (max) & $7.1(1.2)$ & $6.6(1.2)$ & $7.0(1.2)$ & $4.8(0.8)^{a, b, c}$ \\
\hline WMS-R digit span reverse (max) & $5.6(1.3)$ & $4.4(1.4)$ & $5.1(2.0)$ & $3.0(0.7)^{\mathrm{a}}$ \\
\hline D-KEFS Stroop color naming (s) & $32.4(6.4)^{b, d}$ & $49.5(20.8)^{d}$ & $50.3(27.9)^{d}$ & $87.0(6.7)$ \\
\hline D-KEFS Stroop word reading (s) & $23.5(5.7)^{d}$ & $35.9(22.2)^{d}$ & $30.9(19.2)^{d}$ & $85.4(10.3)$ \\
\hline D-KEFS Stroop interference (s) & $56.2(16.9)^{\mathrm{b}, \mathrm{d}}$ & $103.3(47.3)^{d}$ & $82.7(50.5)^{d}$ & $165.0(30.8)$ \\
\hline Letter fluency (F: total) & $18.1(5.7)$ & $7.6(4.4)^{\mathrm{a}}$ & $9.7(7.2)^{\mathrm{a}}$ & $3.5(1.7)^{\mathrm{a}}$ \\
\hline Category fluency (animals: total) & $24.7(5.9)$ & $11.6(6.2)^{a}$ & $6.7(5.4)^{\mathrm{a}}$ & $8.8(3.5)^{\mathrm{a}}$ \\
\hline Trails A (s) & $32.2(5.6)^{\mathrm{b}, \mathrm{d}}$ & $59.5(33.5)$ & $47.0(21.0)$ & $81.7(48.4)$ \\
\hline Trails B (s) & $66.1(20.5)^{b, d}$ & $184.1(89.0)$ & $133.6(110.1)$ & $211.1(94.6)$ \\
\hline \multicolumn{5}{|l|}{ Language skills } \\
\hline WASI vocabulary & $72.2(3.4)$ & $42.6(21.8)^{\mathrm{a}}$ & $34.7(22.7)^{\mathrm{a}}$ & $31.7(13.9)^{a}$ \\
\hline BPVS & $148.5(1.1)$ & $123.8(35.3)^{a}$ & $94.4(49.4)^{a, d}$ & $142.6(10.1)$ \\
\hline GNT & $26.3(2.4)$ & $10.6(9.8)^{a}$ & $2.0(5.3)^{a, b, d}$ & $15.5(6.6)^{\mathrm{a}}$ \\
\hline \multicolumn{5}{|l|}{ Posterior cortical skills } \\
\hline GDA (/24) & $15.8(5.4)$ & $7.8(5.7)^{\mathrm{a}}$ & $11.3(8.3)$ & $5.4(1.9)^{\mathrm{a}}$ \\
\hline VOSP Object Decision (/20) & $19.1(1.6)$ & $15.6(3.0)^{\mathrm{a}}$ & $15.7(5.1)$ & $15.3(4.7)^{\mathrm{a}}$ \\
\hline
\end{tabular}

Mean (SD) scores are shown unless otherwise indicated; maximum scores are shown after tests (in parentheses).

aSignificantly less than controls, ${ }^{b}$ significantly less than bVFTD, ${ }^{c}$ significantly less than SD, dsignificantly less than PNFA (all $p<0.05$ ).

BPVS, British Picture Vocabulary Scale (31); category fluency for animal category and letter fluency for the letter F in 1 min (32); EX, sensitivity to the emotions of others component of the Revised Self-Monitoring Scale (33); GDA, Graded Difficulty Arithmetic (34); GNT, Graded Naming Test (35); MMSE, Mini-Mental State Examination score (36); N/A, not assessed; PAL, Paired Associate Learning test (37); RMT, Recognition Memory Test (38); Stroop D-KEFS, Delis Kaplan Executive System (39); Trails-making task based on maximum time achievable 2.5 min on task A, 5 min on task B (40); VOSP, Visual Object and Spatial Perception Battery (41); WAIS-R, Wechsler Adult Intelligence Scale - Revised (42); WASI,

Wechsler Abbreviated Scale of Intelligence (43); WMS, Wechsler Memory Scale (44).

randomized order, to preclude anticipation or guessing based on previous epochs. For each participant, an interoceptive accuracy index (IA) was calculated based on an established method as follows (3):

$$
\begin{array}{r}
1-\mid \text { actual beats }- \text { reported beats } \mid \\
\quad /((\text { actual beats }+ \text { reported beats }) / 2) .
\end{array}
$$

\section{Emotional Sensitivity Rating}

Patients' caregivers completed the Sensitivity to Socioemotional Expressiveness Score (EX) component of the Revised SelfMonitoring Scale (33), a daily-life index of sensitivity to the emotions of others.

\section{Data Analysis}

Between-group differences were assessed using ANOVAs, except where the homogeneity of variance assumption was violated, when Welch's F test and Games Howell post hoc tests (a multiple comparison procedure without the assumption of homoscedasticity) were used. In addition, we assessed correlations of IA with EX (sensitivity to others' emotions), auditory reverse digit span (a standard index of nonverbal sensory working memory), British Picture Vocabulary score (a standard measure of semantic comprehension), and mean heart rate (a peripheral interoceptive signal characteristic). A threshold $p<0.05$ was accepted as the significance criterion for all tests.

\section{Brain Image Acquisition and Analysis}

Each patient had a sagittal 3-D magnetization-prepared rapidgradient-echo T1-weighted volumetric brain MR sequence (TE/ TR/TI 2.9/2,200/900 ms, dimensions 256256 208, voxel size $1.1 \mathrm{~mm}^{3}$ ), acquired on a Siemens Trio $3 \mathrm{~T}$ MRI scanner using a 32-channel phased-array head-coil. Normalization, segmentation, and modulation of gray and white matter images were 
performed using SPM12 ${ }^{1}$ with default parameter settings and gray matter images were smoothed using a $6 \mathrm{~mm}$ full width-athalf-maximum Gaussian kernel. A study-specific template mean brain image was created by warping all bias-corrected native space brain images to the final DARTEL template and calculating the average of the warped brain images. Total intracranial volume (TIV) was calculated for each patient by summing gray matter, white matter, and cerebrospinal fluid volumes following segmentation of all three tissue classes.

A full factorial model was used to assess associations between IA and regional gray matter volume (voxel intensity) within each syndromic group, incorporating age and TIV as covariates of no interest. Statistical parametric maps of regional gray matter associations were assessed at threshold $p<0.05$ after family-wise error (FWE) correction for multiple voxel-wise comparisons within pre-determined regions of interest [cingulate cortex, insula, and amygdala $(17,18)$ defined from the Harvard-Oxford Brain Atlas]. ${ }^{2}$

\section{RESULTS}

Interoceptive accuracy data and neuroanatomical correlates are presented in Figure 1.

The homogeneity of variance assumption was violated for IA data (Levene's test $p=0.001)$. Welch's $F$ test revealed a main effect of participant group on IA $(p=0.021)$. Games Howell post hoc tests showed that IA was significantly lower in the svPPA group than healthy controls $(p=0.022)$. No other significant group differences were identified for IA. Mean EX was significantly higher in the nfvPPA group than the other patient groups $(p<0.001)$ but did not differ between the bvFTD and svPPA groups $(p=0.29)$. Across the patient cohort, there was a significant positive correlation between IA and $\mathrm{EX}$ ( $\mathrm{rho}=0.516, p=0.004$ ); there was no significant association between IA and reverse digit span (rho $=0.133, p=0.372$ ), British Picture Vocabulary Score $(\mathrm{rho}=0.242, p=0.09)$, mean heart rate $(\mathrm{rho}=0.038, p=0.8)$, age (rho $=-0.062, p=0.67$ ), disease duration ( $\mathrm{rho}=-0.1, p=0.59$ ), or antihypertensive use $(p=0.5)$.

In the svPPA group, IA was significantly positively associated with gray matter volume in right amygdala, right anterior, and posterior cingulate cortex and right insula (all $p<0.05_{\mathrm{FWE}}$ within pre-specified regions of interest). No significant gray matter associations were identified at the prescribed threshold in the other patient groups.

\section{DISCUSSION}

Our findings demonstrate that interoceptive accuracy is impaired in svPPA relative to healthy older individuals. There was a wide range of IA scores in the control group, as typically found in studies of healthy individuals $(3,13)$. Overall performance in the control group was lower than typically found in studies of younger subjects, with several being unable to detect heartbeats, but this is consistent with evidence that interoception declines

${ }^{1}$ www.fil.ion.ucl.ac.uk/spm.

${ }^{2}$ http://fsl.fmrib.ox.ac.uk/fsl/fslwiki/Atlases.

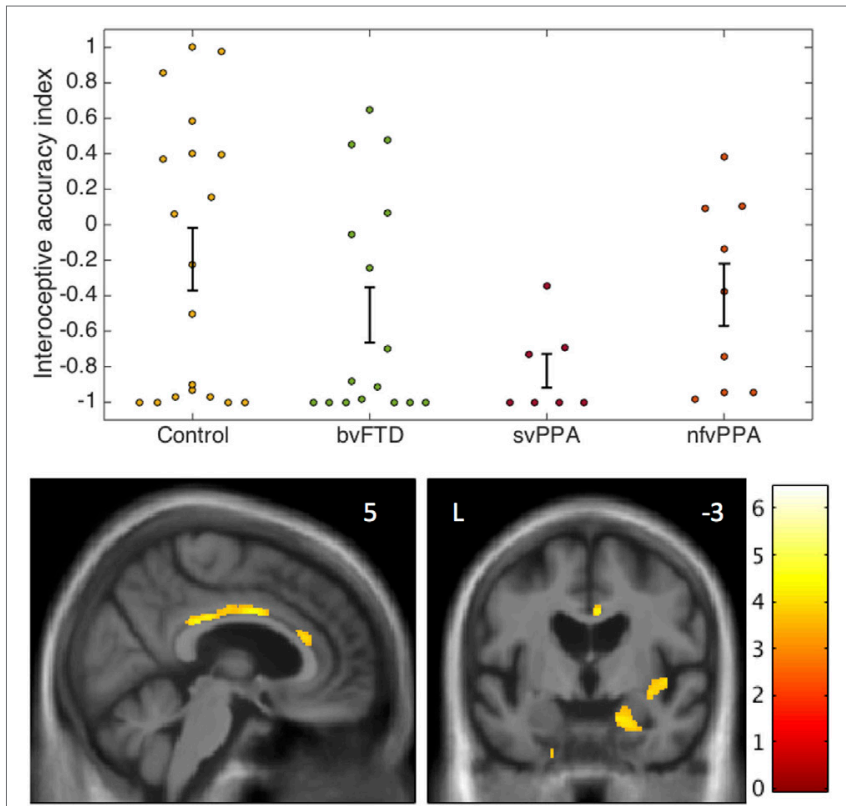

FIGURE 1 | Interoceptive accuracy in participant groups: behavioral and voxel-based morphometry data. The plots (above) show individual raw data for accuracy on the heartbeat counting task expressed as an interoceptive accuracy index (see text) in each participant group. Error bars represent SEM. The statistical parametric map of regional gray matter volume associated with interoceptive accuracy in the impaired svPPA group (below) has been overlaid on representative sections of the normalized study-specific T1-weighted group mean brain MR image; the MNI coordinate (millimeters) of the plane of each section is indicated. The color barcodes $T$ values; the SPM is thresholded here at $p<0.001$ uncorrected over the whole brain for display purpose. Regional local maxima were significant at $p<0.05_{\text {FWE }}$ corrected for multiple comparisons over the whole brain (right amygdala, MNI coordinates [18 -15 -21]) or within pre-specified anatomical regions of interest (anterior cingulate cortex [4 0 34]; posterior cingulate cortex [2 -30 28]; right insula [44 -4 -3]). bvFTD, patients with behavioral variant frontotemporal dementia; Control, healthy control group; L, left; nfvPPA, patients with nonfluent variant primary progressive aphasia; svPPA, patients with semantic variant primary progressive aphasia.

with age (45-47). Over the patient cohort, impaired IA did not correlate with any reduction in generic sensory monitoring, semantic capacity, or peripheral interoceptive signal. In line with current models of interoception $(3,17,18)$ and evidence for abnormal processing of homeostatic and affective signals in FTD syndromes (21), the findings suggest that svPPA affects the initial cognitive decoding of interoceptive signals. Interoceptive accuracy in the patient cohort was correlated with sensitivity to others' emotions: coupled with evidence in the healthy brain (3, $8-10,17,18)$, this suggests that degraded inference of others' emotions from one's own embodied responses might serve as a generic mechanism for the blunted emotional reactivity and empathy loss that characterizes FTD and may be particularly pervasive in svPPA (20). Moreover, interoceptive impairment is a plausible mechanism for the severe impoverishment of selfprojection described in svPPA, and for the increased dependency on exteroceptive signals found in these patients $(24,27)$.

Emotional sensitivity was comparably reduced in both the svPPA and bvFTD groups (relative to the nfvPPA group) here, 
while bvFTD has been associated with impaired interoceptive awareness in previous work (26). Taken together with the present findings, the emerging picture suggests a complex stratification of autonomic abnormalities across FTD syndromes: autonomic reactivity in these syndromes may be differentially altered under particular conditions (such as detection of salient changes in self or environment versus monitoring of bodily states) $(21,26)$. FTD syndromes may target separable levels of interoceptive processing, svPPA producing a more fundamental deficit of interoceptive signal analysis and decoding of autonomic responses to emotion, while bvFTD impairs autonomic reactivity and the metacognitive analysis of body state representations in self and others $(22,48,49)$. The neuroanatomical substrate for impaired interoceptive accuracy in the present svPPA group comprised a rightward-asymmetric cingulo-insulo-amygdalar network: this network is encompassed by the distributed atrophy profile of svPPA (28), has been previously implicated in interoception both in the healthy brain and disease states $(17,18,26)$, and is well-placed anatomically to integrate homeostatic and external socioemotional signals in building representations of self and others (5).

This small study provides proof of principle for further systematic investigation of interoception as an attractive, novel paradigm for deconstructing complex deficits of emotional reactivity, empathy, and self-awareness in neurodegenerative syndromes. At present, we lack quantifiable metrics for cardinal socioemotional symptoms of dementia. Interoception may plausibly underpin such symptoms and can be assessed using simple, objectively verifiable procedures. Clearly, the variation in intrinsic interoceptive sensitivity among healthy people will need to be taken into account in applying interoceptive measures in clinical settings. However, acknowledging this caveat, interoceptive sensitivity warrants further evaluation, both as a potential biomarker in individuals with retained baseline capacity to perform the task and to identify neuroanatomical and physiological correlates, which might yield outcome measures in clinical trials. Future work should assess different interoceptive dimensions longitudinally, in larger cohorts sampling representatively across syndromes and with molecular correlation, to determine the reliability, sensitivity, and specificity of potential interoceptive biomarkers. Larger studies with greater power may additionally reveal less profound interoceptive deficits within the heterogeneous bvFTD population. Control conditions involving exteroceptive counting tasks of comparable difficulty

\section{REFERENCES}

1. Critchley Hugo D, Harrison Neil A. Visceral influences on brain and behavior. Neuron (2013) 77(4):624-38. doi:10.1016/j.neuron.2013.02.008

2. Schandry R. Heart beat perception and emotional experience. Psychophysiology (1981) 18(4):483-8. doi:10.1111/j.1469-8986.1981.tb02486.x

3. Garfinkel SN, Seth AK, Barrett AB, Suzuki K, Critchley HD. Knowing your own heart: distinguishing interoceptive accuracy from interoceptive awareness. Biol Psychol (2015) 104:65-74. doi:10.1016/j.biopsycho.2014.11.004

4. Seth AK, Friston KJ. Active interoceptive inference and the emotional brain. Philos Trans R Soc Lond B Biol Sci (2016) 371:1708. doi:10.1098/rstb.2016.0007

5. Craig AD. How do you feel - now? The anterior insula and human awareness. Nat Rev Neurosci (2009) 10(1):59-70. doi:10.1038/nrn2555

6. Brewer R, Cook R, Bird G. Alexithymia: a general deficit of interoception. $R$ Soc Open Sci (2016) 3:10. doi:10.1098/rsos.150664 might help to further disambiguate interoceptive deficits from other cognitive difficulties impairing task performance. The use of passive interoception tasks such as those based on stimulus timing in the cardiac cycle and measurement of heartbeat-evoked potentials would also be of value to provide further confirmation that deficits in interoceptive reporting are not confounded by other neuropsychological impairments $(12,50)$.

\section{ETHICS STATEMENT}

This study was carried out in accordance with the recommendations of The National Hospital for Neurology and Neurosurgery and Institute of Neurology Joint Research Ethics Committee with written informed consent from all subjects. All subjects gave written informed consent in accordance with the Declaration of Helsinki. The protocol was approved by The National Hospital for Neurology and Neurosurgery and Institute of Neurology Joint Research Ethics Committee.

\section{AUTHOR CONTRIBUTIONS}

Conception and design of the study: CM, JK, and JW. Acquisition and analysis of data: CM, CH, LR, CC, KD, EB, CM, JS, JR, JK, and JW. Drafting of manuscript: CM, CH, RB, JS, JK, and JW.

\section{ACKNOWLEDGMENTS}

We are grateful to all participants for their involvement. The Dementia Research Centre is supported by Alzheimer's Research UK, the Brain Research Trust and the Wolfson Foundation. This work was funded by the Alzheimer's Society, Leonard Wolfson Experimental Neurology Centre, Wellcome Trust, Medical Research Council UK, and the NIHR UCLH Biomedical Research Centre Queen Square Dementia Biomedical Research Unit. CRM is supported by a Clinical Research Fellowship from the Leonard Wolfson Experimental Neurology Centre. $\mathrm{CH}$ and RB hold MRC PhD studentships. CNC was supported by The National Brain Appeal-Frontotemporal Dementia Research Fund. JR is supported by an MRC Clinician Scientist Fellowship (MR/M008525/1) and has received funding from the NIHR Rare Disease Translational Research Collaboration. JW was supported by a Wellcome Trust Senior Clinical Fellowship (Grant No 091673/Z/10/Z).

7. Ondobaka S, Kilner J, Friston K. The role of interoceptive inference in theory of mind. Brain Cogn (2017) 112:64-8. doi:10.1016/j.bandc.2015.08.002

8. Terasawa Y, Moriguchi Y, Tochizawa S, Umeda S. Interoceptive sensitivity predicts sensitivity to the emotions of others. Cogn Emot (2014) 28(8):1435-48. doi:10.1080/02699931.2014.888988

9. Shah P, Catmur C, Bird G. From heart to mind: linking interoception, emotion, and theory of mind. Cortex (2017) 93:220-3. doi:10.1016/j.cortex.2017.02.010

10. Fukushima H, Terasawa Y, Umeda S. Association between interoception and empathy: evidence from heartbeat-evoked brain potential. Int J Psychophysiol (2011) 79(2):259-65. doi:10.1016/j.ijpsycho.2010.10.015

11. Gray MA, Beacher FD, Minati L, Nagai Y, Kemp AH, Harrison NA, et al. Emotional appraisal is influenced by cardiac afferent information. Emotion (2012) 12(1):180-91. doi:10.1037/a0025083

12. Garfinkel SN, Minati L, Gray MA, Seth AK, Dolan RJ, Critchley HD. Fear from the heart: sensitivity to fear stimuli depends on individual 
heartbeats. J Neurosci (2014) 34(19):6573-82. doi:10.1523/jneurosci.350713.2014

13. Tsakiris M, Jiménez AT, Costantini M. Just a heartbeat away from one’s body: interoceptive sensitivity predicts malleability of body-representations. Proc Biol Sci (2011) 278(1717):2470. doi:10.1098/rspb.2010.2547

14. Park HD, Bernasconi F, Bello-Ruiz J, Pfeiffer C, Salomon R, Blanke O. Transient modulations of neural responses to heartbeats covary with bodily self-consciousness. J Neurosci (2016) 36(32):8453-60. doi:10.1523/jneurosci. 0311-16.2016

15. Filippetti ML, Tsakiris M. Heartfelt embodiment: changes in body-ownership and self-identification produce distinct changes in interoceptive accuracy. Cognition (2016) 159:1-10. doi:10.1016/j.cognition.2016.11.002

16. Allen M, Frank D, Schwarzkopf DS, Fardo F, Winston JS, Hauser TU, et al. Unexpected arousal modulates the influence of sensory noise on confidence. Elife (2016) 5:e18103. doi:10.7554/eLife.18103

17. Critchley HD, Wiens S, Rotshtein P, Ohman A, Dolan RJ. Neural systems supporting interoceptive awareness. Nat Neurosci (2004) 7(2):189-95. doi:10.1038/nn1176

18. Garfinkel SN, Critchley HD. Threat and the body: how the heart supports fear processing. Trends Cogn Sci (2016) 20(1):34-46. doi:10.1016/j.tics.2015.10.005

19. Mather M. The emotion paradox in the aging brain. Ann N Y Acad Sci (2012) 1251:33-49. doi:10.1111/j.1749-6632.2012.06471.x

20. Rankin KP, Kramer JH, Miller BL. Patterns of cognitive and emotional empathy in frontotemporal lobar degeneration. Cogn Behav Neurol (2005) 18(1):28-36. doi:10.1097/01.wnn.0000152225.05377.ab

21. Fletcher PD, Nicholas JM, Shakespeare TJ, Downey LE, Golden HL, Agustus JL, et al. Physiological phenotyping of dementias using emotional sounds. Alzheimers Dement (Amst) (2015) 1(2):170-8. doi:10.1016/j.dadm. 2015.02.003

22. Guo CC, Sturm VE, Zhou J, Gennatas ED, Trujillo AJ, Hua AY, et al. Dominant hemisphere lateralization of cortical parasympathetic control as revealed by frontotemporal dementia. Proc Natl Acad Sci U S A (2016) 113:E2430-9. doi:10.1073/pnas.1509184113

23. Downey LE, Fletcher PD, Golden HL, Mahoney CJ, Agustus JL, Schott JM, et al. Altered body schema processing in frontotemporal dementia with C9ORF72 mutations. J Neurol Neurosurg Psychiatry (2014) 85:1016-23. doi:10.1136/jnnp-2013-306995

24. Fletcher PD, Downey LE, Golden HL, Clark CN, Slattery CF, Paterson RW, et al. Pain and temperature processing in dementia: a clinical and neuroanatomical analysis. Brain (2015) 138:3360-72. doi:10.1093/brain/awv276

25. Hsieh S, Irish M, Daveson N, Hodges JR, Piguet O. When one loses empathy: its effect on carers of patients with dementia. J Geriatr Psychiatry Neurol (2013) 26(3):174-84. doi:10.1177/0891988713495448

26. García-Cordero I, Sedeño L, de la Fuente L, Slachevsky A, Forno G, Klein F, et al. Feeling, learning from and being aware of inner states: interoceptive dimensions in neurodegeneration and stroke. Philos Trans R Soc Lond B Biol Sci (2016) 371:20160006. doi:10.1098/rstb.2016.0006

27. Irish M, Piolino P. Impaired capacity for prospection in the dementias theoretical and clinical implications. Br J Clin Psychol (2016) 55(1):49-68. doi:10.1111/bjc.12090

28. Rosen HJ, Gorno-Tempini ML, Goldman WP, Perry RJ, Schuff N, Weiner $\mathrm{M}$, et al. Patterns of brain atrophy in frontotemporal dementia and semantic dementia. Neurology (2002) 58(2):198-208. doi:10.1212/WNL.58.2.198

29. Rascovsky K, Hodges JR, Knopman D, Mendez MF, Kramer JH, Neuhaus J, et al. Sensitivity of revised diagnostic criteria for the behavioural variant of frontotemporal dementia. Brain (2011) 134(Pt 9):2456-77. doi:10.1093/ brain/awr179

30. Gorno-Tempini ML, Hillis AE, Weintraub S, Kertesz A, Mendez M, Cappa SF, et al. Classification of primary progressive aphasia and its variants. Neurology (2011) 76(11):1006-14. doi:10.1212/WNL.0b013e31821103e6
31. Dunn LM, Whetton C. British Picture Vocabulary Scale. Windsor, England: NFER-Nelson (1982).

32. Gladsjo JA, Schuman CC, Evans JD, Peavy GM, Miller SW, Heaton RK. Norms for letter and category fluency: demographic corrections for age, education, and ethnicity. Assessment (1999) 6(2):147-78. doi:10.1177/107319119900600204

33. Lennox RD, Wolfe RN. Revision of the self-monitoring scale. J Pers Soc Psychol (1984) 46(6):1349-64. doi:10.1037/0022-3514.46.6.1349

34. Jackson M, Warrington EK. Arithmetic skills in patients with unilateral cerebral lesions. Cortex (1986) 22(4):611-20.

35. McKenna P, Warrington EK. Testing for nominal dysphasia. J Neurol Neurosurg Psychiatry (1980) 43(9):781-8.

36. Folstein MF, Folstein SE, McHugh PR. "Mini-mental state". A practical method for grading the cognitive state of patients for the clinician. J Psychiatr Res (1975) 12(3):189-98.

37. Warrington E. The Camden Memory Test Battery. Brighton, UK: Psychology Press (1996).

38. Warrington EK. Recognition Memory Test: Rmt.(Words). Test Booklet 1: NFER-Nelson Publishing Company (1984).

39. Delis DC, Kaplan E, Kramer JH. Delis-Kaplan Executive Function System (D-KEFS). Psychological Corporation (2001).

40. Lezak MD. Neuropsychological Assessment. USA: Oxford University Press (2004).

41. Warrington EK, James M. The Visual Object and Space Perception Battery. Bury St Edmunds, England: Thames Valley Test Company (1991).

42. Wechsler D, De Lemos MM. Wechsler Adult Intelligence Scale-Revised. Harcourt Brace Jovanovich (1981).

43. Wechsler D. WAIS-III: Wechsler Adult Intelligence Scale. Psychological Corporation (1997).

44. Wechsler D. Wechsler Memory Scale-Revised (WMS-R). Psychological Corporation (1987).

45. Khalsa SS, Rudrauf D, Tranel D. Interoceptive awareness declines with age. Psychophysiology (2009) 46(6):1130-6. doi:10.1111/j.1469-8986.2009.00859.x

46. Murphy J, Brewer R, Catmur C, Bird G. Interoception and psychopathology: a developmental neuroscience perspective. Dev Cogn Neurosci (2017) 23: 45-56. doi:10.1016/j.dcn.2016.12.006

47. Murphy J, Geary H, Millgate E, Catmur C, Bird G. Direct and indirect effects of age on interoceptive accuracy and awareness across the adult lifespan. Psychon Bull Rev (2017):1-10. doi:10.3758/s13423-017-1339-Z

48. Balconi M, Cotelli M, Brambilla M, Manenti R, Cosseddu M, Premi E, et al. Understanding emotions in frontotemporal dementia: the explicit and implicit emotional cue mismatch. J Alzheimers Dis (2015) 46:211-25. doi:10.3233/ jad- 142826

49. Joshi A, Mendez MF, Kaiser N, Jimenez E, Mather M, Shapira JS. Skin conductance levels may reflect emotional blunting in behavioral variant frontotemporal dementia. J Neuropsychiatry Clin Neurosci (2014) 26(3):227-32. doi:10.1176/appi.neuropsych.12110332

50. Maister L, Tang T, Tsakiris M. Neurobehavioral evidence of interoceptive sensitivity in early infancy. Elife (2017) 6:e25318. doi:10.7554/eLife.25318

Conflict of Interest Statement: The authors declare that the research was conducted in the absence of any commercial or financial relationships that could be construed as a potential conflict of interest.

Copyright (c) 2017 Marshall, Hardy, Russell, Clark, Dick, Brotherhood, Bond, Mummery, Schott, Rohrer, Kilner and Warren. This is an open-access article distributed under the terms of the Creative Commons Attribution License (CC BY). The use, distribution or reproduction in other forums is permitted, provided the original author(s) or licensor are credited and that the original publication in this journal is cited, in accordance with accepted academic practice. No use, distribution or reproduction is permitted which does not comply with these terms. 